\title{
Direct thrombectomy versus bridging thrombolysis with mechanical thrombectomy in middle cerebral artery stroke: a real-world analysis through National Inpatient Sample data
}

\author{
Sandeep Kandregula, MBBS, MCh, ${ }^{1}$ Amey R. Savardekar, MD, ${ }^{1}$ Pankaj Sharma, MD, ${ }^{2}$ \\ Jerry McLarty, PhD, ${ }^{3}$ Jennifer Kosty, MD, ${ }^{1}$ Krystle Trosclair, PhD, ${ }^{1}$ Hugo Cuellar, MD, PhD, ${ }^{4}$ and \\ Bharat Guthikonda, MD1
}

Departments of ${ }^{1}$ Neurosurgery, ${ }^{2}$ Neurology, and ${ }^{4}$ Radiology, Ochsner LSU Health Shreveport; and ${ }^{3}$ Department of Oncology, Feist-Weiller Cancer Center, Ochsner LSU Health Shreveport, Louisiana

\begin{abstract}
OBJECTIVE A paradigm shift in the management of acute ischemic stroke (AIS) due to large-vessel occlusion (LVO) occurred after 2015 when 7 randomized controlled trials demonstrated better outcomes using second-generation thrombectomy devices combined with best medical management than did stand-alone intravenous thrombolysis (IVT) with tissue plasminogen activator (tPA). All recently published landmark trials were designed to study the outcome of mechanical thrombectomy (MT); therefore, the majority of the patients enrolled in these trials received intravenous tPA. Currently, initiating IVT before MT is a matter of debate. Recent trials (DIRECT-MT, DEVT) exploring this clinical question showed noninferiority of MT alone compared with the combined treatment. With this uncertainty, the authors aimed to explore real-world data through the latest National Inpatient Sample (NIS) to compare the safety and outcomes of MT alone with bridging IVT and MT in AIS due to LVO in the middle cerebral artery (MCA).
\end{abstract}

METHODS NIS data from 2017 to 2018 were analyzed to compare the outcomes and safety profiles of patients who underwent MT+IVT with those who underwent MT alone.

RESULTS A total of 2895 patients were included in the final analysis (MT, $n=1669 ;$ MT+IVT, $n=1226$ ). The mean National Institutes of Health Stroke Scale score was 16.2 (SD 6.1) in the MT group and 16.6 (SD 5.97) in the MT+IVT group $(p=0.04)$. With respect to comorbidities, the two groups did not differ in rates of hypertension $(p=0.730)$, atrial fibrillation/flutter $(p=0.828)$, and smoking status $(p=0.914)$. The rate of diabetes mellitus was significantly higher in the MT group $(28 \%)$ than in the MT+IVT group $(22.1 \%)(p<0.001)$. The frequency of intracerebral hemorrhage $(I C H)$ in the MT group was $17.7 \%(n=296)$ and $21.5 \%(n=263)$ in the MT+IVT group $(p=0.012)$. Intraventricular hemorrhage $(p=$ $0.875)$, subarachnoid hemorrhage $(p=0.99)$, and vasospasm $(p=0.976)$ did not differ significantly between the groups. The primary outcome considered was disability status between the groups; $23.8 \%$ of patients in the MT+IVT group had minimal disability versus $18.2 \%$ in the MT group $(p=0.001)$. The risk of progressing to severe disability from minimal disability decreased with the addition of IVT to MT (OR $0.762,95 \% \mathrm{Cl} 0.637-0.912)$. The adjusted odds ratio for ICH in the MT+IVT group was $1.28(95 \% \mathrm{Cl} 1.043-1.571, p=0.018)$ and $2.676(95 \% \mathrm{Cl} 1.259-5.686, p=0.01)$ for access-site hemorrhages.

CONCLUSIONS In the analysis of the NIS database, the MT+IVT group had significantly higher rates of minimal disability at the time of hospital discharge versus the MT-alone group, despite a higher rate of $\mathrm{ICH}$. The question of whether to treat patients with MT+IVT rather than MT alone is currently being addressed in ongoing prospective clinical trials (SWIFT-DIRECT [NCT03494920], MR CLEAN-NO IV [ISRCTN80619088], and DIRECT-SAFE [NCT03494920]). The results of these studies will contribute to greater understanding and progressive improvement in outcomes for AIS patients.

https://thejns.org/doi/abs/10.3171/2021.4.FOCUS21132

KEYWORDS mechanical thrombectomy; intravenous thrombolysis; intracerebral hemorrhage; atrial fibrillation; hypertension 
$\mathrm{T}$ HE global burden of stroke is a persistent and escalating problem. It is estimated that $4 \%$ of US adults will have had a stroke by 2030 , increasing the direct annual costs from $\$ 71.55$ billion to $\$ 186$ billion (USD). ${ }^{1,2}$ Approximately $85 \%$ of all strokes are ischemic, and large-vessel occlusion (LVO) accounts for half of them. ${ }^{3}$ A paradigm shift in the management of acute ischemic stroke (AIS) due to LVO occurred after 2015 when 7 randomized controlled trials demonstrated better outcomes using second-generation thrombectomy devices combined with best medical management than did stand-alone intravenous thrombolysis (IVT) with tissue plasminogen activator (tPA). ${ }^{4-10}$ The advantages of IVT include early recanalization before mechanical thrombectomy (MT), thus obviating the need for MT; altering the thrombus composition, thus facilitating MT; and dissolving thrombotic debris in downstream vessels, thus facilitating reperfusion. Despite the potential benefit of intravenous tPA in achieving recanalization, there is a potential increased risk of systemic and intracranial hemorrhage. All recently published landmark trials were designed to study the outcome of MT, and therefore the majority of the patients enrolled in these trials received intravenous tPA. The pooled post hoc analysis of major trials did not show better recanalization rates for IVT combined with MT. ${ }^{11}$ Currently, initiating IVT before MT is a matter of debate. Recent trials (DIRECT-MT, DEVT) exploring this clinical question showed noninferiority of MT alone compared with the combined treatment. ${ }^{12,13}$ The DIRECT-MT trial was conducted in China, and the applicability of those results to the US population needs to be studied further. Also, the sample size was small, and prespecified noninferiority margins were generous. The SKIP trial also showed similar outcomes between MT and combined treatment, although it failed to meet the noninferiority margin. ${ }^{14}$ With this uncertainty, we aimed to explore the real-world data through the latest National Inpatient Sample (NIS) to compare the safety and outcomes of MT alone with bridging IVT and MT in AIS because of an LVO in the middle cerebral artery (MCA). The limitations in using the NIS database for this study are that the data do not provide the time of onset of stroke to hospitalization or an objective scale of functional status at discharge, or whether each case met eligibility criteria for IVT. Acknowledging these limitations, we explored the NIS data to provide meaningful analysis to answer the above clinical question.

\section{Methods}

\section{Study Population}

Data were extracted from the NIS database for 2017 to 2018; this database is the largest publicly available allpayer database in the US (https://www.hcup-us.ahrq.gov/ nisoverview.jsp). The unweighted data contain more than 7 million hospitalizations. These were coded in the form of diagnostic and procedural ICD-10 codes. Inclusion criteria for the study were 1) MCA stroke as the primary cause of admission, 2) the presence of National Institutes of Health Stroke Scale (NIHSS) score in the form of an ICD-10 diagnostic code, 3 ) age $>18$ years, 4 ) an NIHSS score $\geq 5$ and $\leq$ 30 , and 5) patients who underwent MT alone or MT+IVT.

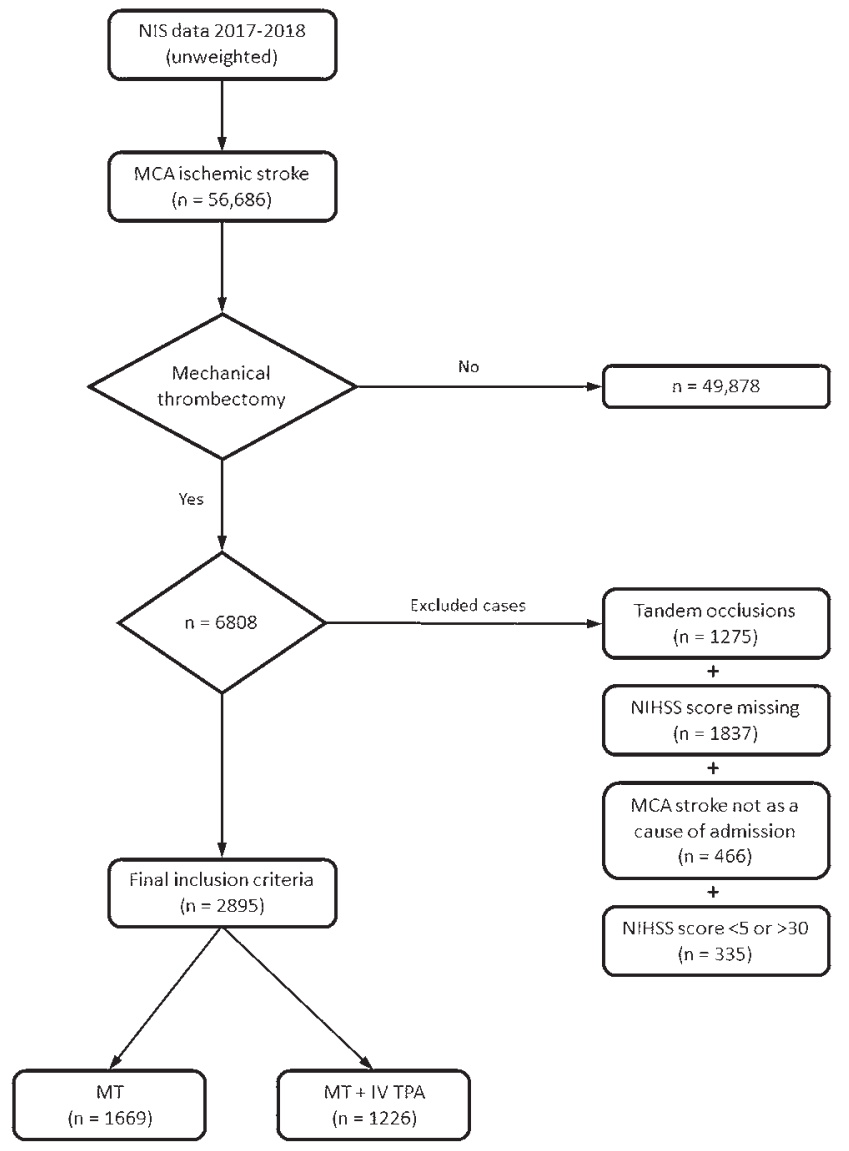

FIG. 1. Flow diagram showing inclusion and exclusion of the cases.

Patients were excluded from the study if 1) there was the presence of a tandem LVO, 2) an MCA stroke was not a primary cause of admission, 3) there was an absence of an NIHSS score or score $<5$ or $>30$, or 4 ) the patient did not undergo MT as the primary treatment procedure. Detailed inclusion and exclusion criteria are shown in Fig. 1.

\section{Data Extraction and Patient Characteristics}

The data were extracted from the NIS data (unweighted) based on the ICD-10 diagnostic and procedural codes. Following the adoption of ICD-10 data, the granularity of the diagnostic coding was significantly increased, giving the chance to explore more entities for the analysis. The codes used for the diagnosis of MCA stroke, internal carotid artery stroke, NIHSS score, presence of intracerebral hemorrhage $(\mathrm{ICH})$, intraventricular hemorrhage (IVH), subarachnoid hemorrhage (SAH), hypertension, smoking status, atrial fibrillation/atrial flutter, diabetes, access-site hemorrhage, vasospasm, IVT injection (same hospital/elsewhere), ventilation status (< 24, 24-96, and $>96$ hours), and contrast dye allergy are provided in Supplementary Table 1. The data were grouped based on the intervention (MT alone vs MT+IVT). Patient socioeconomic characteristics such as patient location (urban/ rural), hospital location, patient zip code, and median income were also analyzed. As the NIS data did not provide any objective scale for measuring stroke severity, like the 
TABLE 1. Demographics and disability status of the MT and MT+IVT patient groups

\begin{tabular}{|c|c|c|c|}
\hline Variable & MT $(n=1669)$ & MT+IVT $(n=1226)$ & $\mathrm{p}$ Value \\
\hline Mean age, yrs & $69.9(14.8)$ & $69.23(4.4)$ & 0.09 \\
\hline Sex & & & 0.96 \\
\hline M & $765(45.8)$ & $563(45.9)$ & \\
\hline $\mathrm{F}$ & $904(54.2)$ & $663(54.1)$ & \\
\hline Diabetes & $467(28)$ & $271(22.1)$ & $<0.001$ \\
\hline Hypertension & $1117(66.9)$ & $828(67.5)$ & 0.730 \\
\hline Atrial fibrillation/flutter & $671(40.2)$ & $488(39.8)$ & 0.828 \\
\hline Smoking & $575(34.5)$ & $420(34.3)$ & 0.914 \\
\hline Mean NIHSS score & $16.2(6.1)$ & $16.6(5.97)$ & 0.04 \\
\hline \multicolumn{4}{|l|}{ Race } \\
\hline White & $1117(69.7)$ & $807(68.9)$ & \\
\hline Black & $263(16.4)$ & $167(14.3)$ & \\
\hline Hispanic & $124(7.7)$ & $110(9.4)$ & \\
\hline Asian/Pacific Islander & $41(2.6)$ & $39(3.3)$ & \\
\hline Native American & $5(0.3)$ & $4(0.3)$ & \\
\hline Other & $53(3.3)$ & $44(3.8)$ & \\
\hline Missing & $66(3.95)$ & $55(4.4)$ & \\
\hline \multicolumn{4}{|l|}{ Discharge destination } \\
\hline Routine & $304(18.2)$ & $292(23.8)$ & \\
\hline Transfer to short-term hospital & $49(2.9)$ & $41(3.3)$ & \\
\hline Transfer to other* & $991(59.4)$ & $658(53.7)$ & \\
\hline Home healthcare & $174(10.4)$ & $122(10)$ & \\
\hline Against medical advice & $5(0.3)$ & $3(0.2)$ & \\
\hline Died & $144(8.6)$ & $109(8.9)$ & \\
\hline \multicolumn{4}{|c|}{ Disability based on discharge destination } \\
\hline Minimal disability & $304(18.2)$ & $292(23.8)$ & 0.001 \\
\hline Moderate to severe disability & $1219(73.1)$ & $824(67.3)$ & \\
\hline Died & $144(8.6)$ & $109(8.9)$ & \\
\hline Mean hospital charges, USD & $\$ 173,544.32(\$ 133,158.19)$ & $\$ 184,908.22(\$ 132,582.25)$ & $<0.001$ \\
\hline
\end{tabular}

Values represent the number of patients (\%) or the mean (SD) unless stated otherwise. Boldface type indicates statistical significance.

* Hospital, short-term skilled facility, or hospice care.

modified Rankin Scale or postprocedural perfusion status (Thrombolysis in Cerebral Infarction), the current study considered disability status as its primary outcome. Disability status was defined based on the discharge disposition and grouped into mild, moderate to severe, and inhospital death. Discharge to home was considered a mild disability; discharge to any other facility (hospital, shortterm skilled facility, or hospice care) was considered moderate to severe disability. Patients who died during hospital admission were categorized as a third discharge category. Secondary outcomes analyzed were the presence of ICH, IVH, SAH, vasospasm, and access-site hemorrhages.

\section{Statistical Analysis}

All variables are described in a standard descriptive fashion. Continuous variables are described as mean (SD), median as appropriate, and categorical variables as frequencies. Groups were compared using the chi-square test for categorical variables, the t-test, and the Mann-Whitney U-test for continuous variables where applicable. Ordinal regression analysis was performed to predict the factors affecting the primary outcome (disability status) after adjusting for all the covariates and binary regression analysis for the secondary outcomes. Statistical significance was considered if the $p$ value (two-tailed) was $<0.05$. Statistical analysis was performed using IBM SPSS version 27 (IBM Corp.). Odds ratio and 95\% confidence interval were derived and are presented for all outcomes after adjusting for the potential confounding factors. The intervention's effect was presented for the secondary outcomes after adjusting for covariate factors.

\section{Results \\ Clinical Characteristics}

A total of 2895 patients were included in the final analysis (MT, $\mathrm{n}=1669$; and MT+IVT, $\mathrm{n}=1226$ ). The mean ages were 69.9 years (SD 14.8 years) in the MT group and 69.23 years (SD 14.4 years) in the MT+IVT group (p $=0.09$ ) (Table 1). The male-to-female ratio between the groups was not statistically significant (MT, $45.8 \%$ and 


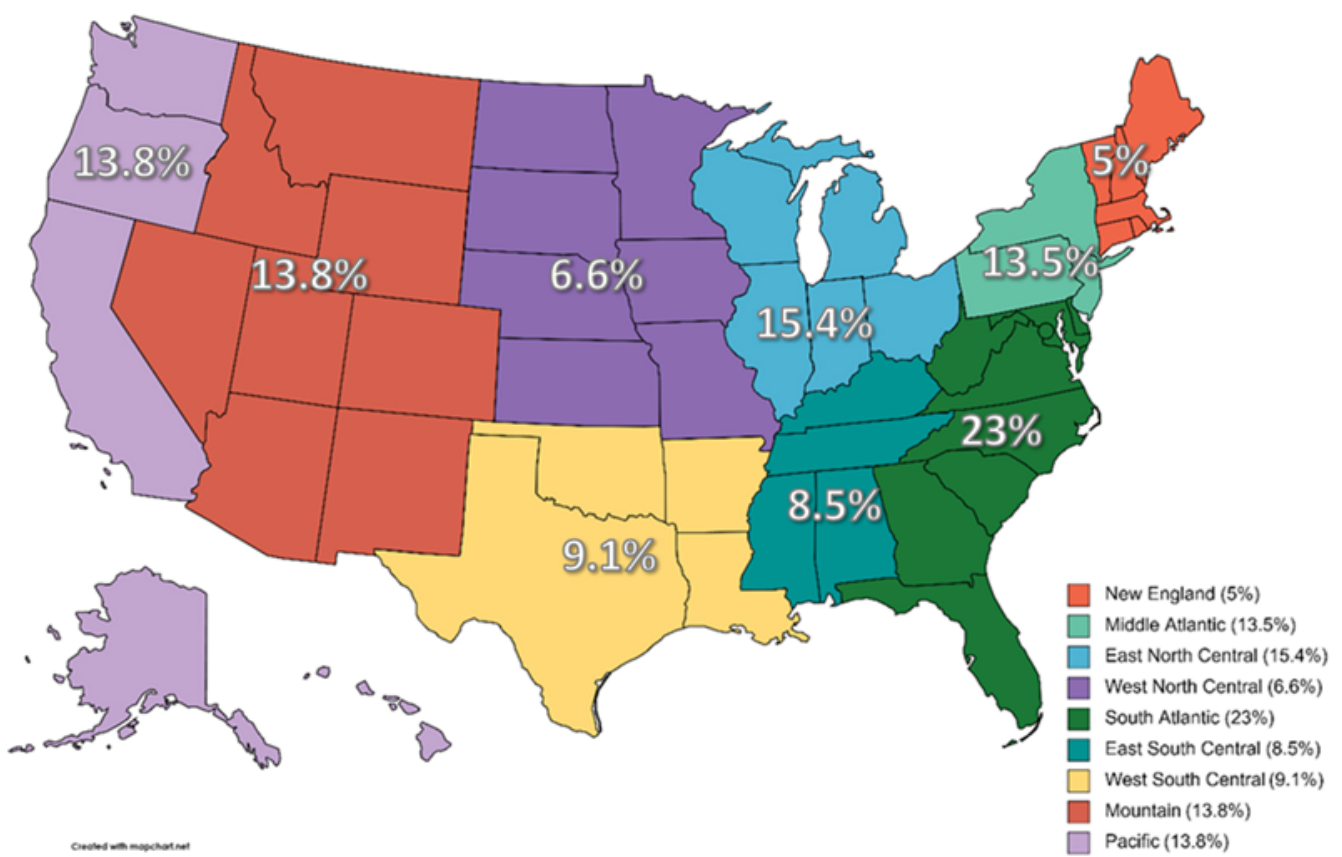

FIG. 2. Distribution of patient cohort based on hospital divisions across the US. Created using mapchart.net. CC BY-SA 4.0 (https://creativecommons.org/licenses/by-sa/4.0/).

54.2\%; and MT+IVT, 45.9\% and 54.1\%) $(\mathrm{p}=0.96)$. White (MT, 69.7\%; and MT+IVT, 68.9\%) and Black (MT, 16.4\%; and MT+IVT, $14.3 \%$ ) were the predominant race categories. The mean NIHSS score was 16.2 (SD 6.1) in the MT group and 16.6 (SD 5.97) in the MT+IVT group ( $p=0.04$ ). With respect to comorbidities, the two groups did not differ in rates of hypertension ( $\mathrm{p}=0.730)$, atrial fibrillation/ flutter $(\mathrm{p}=0.828)$, and smoking status $(\mathrm{p}=0.914)$. Diabetes was significantly higher in the MT group (28\%) versus the MT+IVT group $(22.1 \%)(\mathrm{p}<0.001)$.

\section{Socioeconomic Characteristics}

The most common insurance was Medicare (MT, 64.9\%; and MT+IVT, 61.1\%) in both groups, followed by private insurance (MT, 19.6\%; and MT+IVT, 24.3\%). Patient location was stratified based on the 6-category urban-rural health scheme of the National Center for Health Statistics. Most patients lived in "central" counties of metro areas with a population $\geq 1$ million people (MT, 31.9\%; and MT+IVT, 30.2\%), followed by "fringe" counties of metro areas with a population $\geq 1$ million people (MT, 23.9\%; and MT+IVT, 24.2\%). The South Atlantic region was the most common area (MT, 24.2\%; and MT+IVT, 21\%) (Fig. 2). Complete details of the socioeconomic characteristics are presented in Table 2. Patients in the 0-25th percentile for median household income per zip code were slightly overrepresented compared with the other percentiles (MT, 29.3\%; and MT+IVT, 28.9\%) (p=0.824). The mean hospital charges were $\$ 173,544$ (SD $\$ 133,158.19)$ for the MT group and $\$ 184,908.22$ (SD $\$ 132,582.25$ ) for the MT+IVT group $(\mathrm{p}<0.001)$.

\section{Intervention and Complications}

The frequencies of ICH were $17.7 \%(n=296)$ in the
MT group and $21.5 \%(n=263)$ in the MT+IVT group $(\mathrm{p}$ $=0.012)$. IVH $(p=0.875)$, SAH $(p=0.999)$, and vasospasm $(p=0.976)$ did not differ significantly between the groups. The MT+IVT group (1.7\%) had more access-site hemorrhages compared with the MT group $(0.7 \%)(\mathrm{p}=$ 0.013). Mechanical ventilation status was not significantly different between the groups (Table 3). The mean hospital lengths of stay were 8.17 days (SD 9.91 days) in the MT group and 7.16 days (SD 6.9 days) in the MT+IVT group $(p=0.002)$.

\section{Primary and Secondary Outcomes}

The primary outcome considered was disability status; $23.8 \%$ of patients in the MT+IVT group had minimal disability versus $18.2 \%$ in the MT group $(\mathrm{p}=0.001)$ (Fig. 3). The in-hospital death rate was similar in both groups (MT, 8.6\%; and MT+IVT, 8.9\%). An ordinal regression analysis was performed to calculate the OR and 95\% CI for each independent variable's effect on the outcome (Table 4). The OR for secondary outcomes (IVH, ICH, SAH, vasospasm, and access-site hemorrhages) was calculated through binary regression analysis after adjusting the effect of all independent variables (Table 5). Ordinal regression analysis revealed multiple factors predicting the outcome. The risk of progressing to severe disability from minimal disability decreased with the addition of IVT to MT treatment (OR $0.762,95 \%$ CI $0.637-0.912$ ). The adjusted OR for ICH in the MT+IVT group was $1.28(95 \%$ CI $1.043-1.571, \mathrm{p}=$ $0.018)$ and $2.676(95 \%$ CI $1.259-5.686, \mathrm{p}=0.01)$ for access-site hemorrhages.

\section{Discussion}

In the current study, we found a significantly higher rate 
TABLE 2. Socioeconomic data of the patient cohort

\begin{tabular}{|c|c|c|c|}
\hline Variable & MT & MT+IVT & $p$ Value \\
\hline \multicolumn{4}{|l|}{ Insurance } \\
\hline Medicare & $1082(64.9)$ & 749 (61.1) & \\
\hline Medicaid & $151(9.1)$ & $104(8.5)$ & \\
\hline Private & $327(19.6)$ & $298(24.3)$ & \\
\hline Self-pay & $64(3.8)$ & $56(4.6)$ & \\
\hline No charge & $6(0.4)$ & $4(0.3)$ & \\
\hline Other & $37(2.2)$ & $14(1.1)$ & \\
\hline \multicolumn{4}{|l|}{ Hospital division } \\
\hline New England & $83(5)$ & $63(5.1)$ & \\
\hline Middle Atlantic & $252(15.1)$ & $139(11.3)$ & \\
\hline East North Central & $268(16.1)$ & $177(14.4)$ & \\
\hline West North Central & $98(5.9)$ & $92(7.5)$ & \\
\hline South Atlantic & $408(24.4)$ & $258(21)$ & \\
\hline East South Central & $140(8.4)$ & $105(8.6)$ & \\
\hline West South Central & $131(7.8)$ & $132(10.8)$ & \\
\hline Mountain & $82(4.9)$ & $68(5.5)$ & \\
\hline Pacific & $207(12.4)$ & $192(15.7)$ & \\
\hline \multicolumn{4}{|l|}{ Patient location } \\
\hline $\begin{array}{l}\text { "Central" counties of metro } \\
\text { areas of } \geq 1 \text { million people }\end{array}$ & $529(31.9)$ & $367(30.2)$ & \\
\hline $\begin{array}{l}\text { "Fringe" counties of metro } \\
\text { areas of } \geq 1 \text { million people }\end{array}$ & $397(23.9)$ & $294(24.2)$ & \\
\hline $\begin{array}{l}\text { Counties in metro areas of } \\
250,000-999,999 \text { people }\end{array}$ & $367(22.1)$ & $291(23.9)$ & \\
\hline $\begin{array}{l}\text { Counties in metro areas of } \\
50,000-249,999 \text { people }\end{array}$ & $128(7.7)$ & $107(8.8)$ & \\
\hline Micropolitan counties & $131(7.9)$ & $95(7.8)$ & \\
\hline $\begin{array}{l}\text { Not metropolitan or micro- } \\
\text { politan counties }\end{array}$ & $107(6.4)$ & $62(5.1)$ & \\
\hline Missing & $10(0.59)$ & $10(0.81)$ & \\
\hline \multicolumn{4}{|l|}{$\begin{array}{l}\text { Median household income for } \\
\text { patient zip code, percentile }\end{array}$} \\
\hline $0-25$ th & $480(29.3)$ & $346(28.9)$ & \multirow{4}{*}{0.824} \\
\hline 26th-50th & $426(26)$ & $310(25.9)$ & \\
\hline 51st-75th & $398(24.3)$ & $307(25.6)$ & \\
\hline 76th-100th & $337(20.5)$ & $234(19.5)$ & \\
\hline Missing & $28(1.6)$ & $29(2.3)$ & \\
\hline
\end{tabular}

Values represent the number of patients (\%) unless stated otherwise.

of minimal disability in patients who received MT+IVT versus MT alone for MCA LVO despite higher rates of intracranial hemorrhage. In contrast to these results, the recent DIRECT-MT, ${ }^{13}$ DEVT, ${ }^{12}$ and SKIP ${ }^{14}$ trials all demonstrated noninferiority of MT alone compared with MT+IVT; however, the MT-alone group in our analysis does not include all patients within the 4.5-hour window after stroke onset for IVT. Similar to our study, the SKIP trial demonstrated a higher rate of any ICH (symptomatic and asymptomatic together) in the MT+IVT group (MT $33.7 \%$ vs MT+IVT 50.5\%). After adjusting for all independent variables, the effect of adding IVT to MT reduced the chance of having moderate to severe disability by $34 \%$
TABLE 3. Complications and ventilation of the MT and MT+IVT groups

\begin{tabular}{|c|c|c|c|}
\hline Complication & MT $(n=1669)$ & MT+IVT $(n=1226)$ & $\mathrm{p}$ Value \\
\hline $\mathrm{ICH}$ & $296(17.7)$ & $263(21.5)$ & 0.012 \\
\hline IVH & $22(1.3)$ & $17(1.4)$ & 0.875 \\
\hline $\mathrm{SAH}$ & $98(5.9)$ & $72(5.9)$ & 0.999 \\
\hline Vasospasm & $22(1.3)$ & $16(1.3)$ & 0.976 \\
\hline Access-site hemorrhage & $12(0.7)$ & $21(1.7)$ & 0.013 \\
\hline Contrast allergy & $13(0.8)$ & $10(0.8)$ & 0.912 \\
\hline \multicolumn{4}{|l|}{ Ventilation, hrs } \\
\hline$<24$ & $81(4.9)$ & $65(5.3)$ & 0.586 \\
\hline $24-96$ & $136(8.1)$ & $102(8.3)$ & 0.868 \\
\hline$>96$ & $91(5.5)$ & $71(5.8)$ & 0.695 \\
\hline Mean LOS, days & $8.17(9.91)$ & $7.16(6.9)$ & 0.002 \\
\hline
\end{tabular}

LOS $=$ length of stay.

Values represent the number of patients (\%) or the mean (SD) unless stated otherwise. Boldface type indicates statistical significance.

(OR 0.762). In addition to IVT, an increase in age, presence of ICH, IVH, SAH, vasospasm, diabetes as a comorbidity, and a higher NIHSS score influenced the disability status.

The primary outcome considered in most of the trials was functional independence at 90 days posttreatment (shift of modified Rankin Scale score). In the DEVT trial, $54.3 \%$ of the MT-alone group achieved functional independence compared with $46.6 \%$ in the MT+IVT group. ${ }^{12}$ These findings are in agreement with the DIRECT-MT trial as well. Similarly, in the SKIP trial, $59.4 \%$ of patients achieved functional independence at 90 days in the MTalone group compared with $57.3 \%$ in the MT+IVT group (OR 1.09), a difference that was not significant. ${ }^{14}$ These three trials all used a binary outcome measure, whereas we separated outcome into three different groups, contributing to our different findings.

Secondary outcomes in our study were limited to $\mathrm{ICH}$, access-site hemorrhages, SAH, and vasospasm due to limitations of the NIS database. As previously mentioned, we saw a higher ICH rate in our MT+IVT group, even though this group had a higher rate of minimal disability at discharge. This may be because many of these hemorrhages were not clinically significant, a characteristic that is impossible to determine with our methodology. The DEVT, DIRECT-MT, and SKIP trials all examined symptomatic ICH, finding no significant difference in this measure between the MT-alone and MT+IVT groups..$^{12-14}$ The benefit of both IVT and MT in treating AIS has been established. ${ }^{4-10}$ The addition of bridging IVT to MT has pros and cons. Factors favoring thrombolysis initiation are good collaterals, more distal location of the thrombus, a low NIHSS score, infarcts of multiple territories, and any factor precluding rapid access to the MT. Factors favoring MT are proximal occlusions, use of anticoagulants/ineligibility for thrombolysis, and a higher risk of ICH.

\section{Time as the Most Significant Influence}

Time of intervention after the onset of a stroke is considered the most crucial factor for salvaging the ischemic 
Mechanical thrombectomy and IV TPA

Mechanical thrombectomy

$$
\begin{aligned}
& \begin{array}{llllll}
0 \% & 20 \% & 40 \% & 60 \% & 80 \% & 100 \%
\end{array} \\
& \text { Distribution of disability in MT alone vs MT +IV TPA } \\
& =\text { Minimal disability } \\
& \text { = Moderate to severe }=\text { Death }
\end{aligned}
$$

FIG. 3. Distribution of disability between the MT-alone and MT+IVT groups.

brain. Most trials considered 4.5 to 6 hours for the administration of IVT. Based on the HERMES group's metanalysis ${ }^{15}$ the median time from stroke onset to endovascular perfusion was 196 minutes (IQR 142-267 minutes). After the paradigm shift of stroke management from IVT alone to endovascular thrombectomy and bridging thrombolysis, two further trials, DEFUSE ${ }^{16}$ and DAWN,${ }^{17}$ demonstrated improved functional outcomes in patients who received MT and IVT 6 to 16 hours after symptom onset. The DAWN trial concentrated on the clinical deficit and radiological infarct mismatch, whereas the DEFUSE trial selection was based on perfusion imaging. The results from these trials were promising in the means of care individualization. Unfortunately, the NIS database did not provide data regarding time from symptom onset to treatment, limiting our study. It is possible that the patients in our study in the MT+IVT group had better outcomes than the MT-alone group because the latter may have presented outside of the 4.5-hour window for IVT administration eligibility.

\section{Limitations}

There are several limitations to this study. The most significant limitation, as previously discussed, is the lack of data regarding time from symptom onset to interven-

TABLE 4. Generalized linear regression model for outcomes: ordinal regression analysis for the primary outcome (disability status)

\begin{tabular}{lccc}
\hline \multicolumn{1}{c}{ Factor } & OR & $95 \% \mathrm{Cl}$ & $\mathrm{p}$ Value \\
\hline Age & 1.041 & $1.032-1.050$ & $<0.001$ \\
\hline MT+IVT & 0.762 & $0.637-0.912$ & $\mathbf{0 . 0 0 3 ^ { * }}$ \\
\hline IVH & 2.597 & $1.192-5.657$ & $\mathbf{0 . 0 1 6}$ \\
\hline SAH & 1.708 & $1.168-2.497$ & $\mathbf{0 . 0 0 6}$ \\
\hline Vasospasm & 0.522 & $0.251-1.084$ & 0.081 \\
\hline Diabetes & 1.497 & $1.218-1.841$ & $<0.001$ \\
\hline ICH & 1.974 & $1.553-2.510$ & $<0.001$ \\
\hline Ventilation, hrs & & & \\
\hline$<24$ & 2.553 & $1.670-3.904$ & $<0.001$ \\
\hline 24-96 & 4.829 & $3.462-6.735$ & $<0.001$ \\
\hline$>96$ & 4.768 & $3.143-7.231$ & $<0.001$ \\
\hline NIHSS score & 1.076 & $1.059-1.092$ & $<0.001$ \\
\hline
\end{tabular}

Boldface type indicates statistical significance.

${ }^{*}$ Note the effect of IVT on outcome.
TABLE 5. Binary regression analysis for secondary outcomes focusing on the intervention's effect on outcome after considering all covariate factors

\begin{tabular}{lcccc}
\hline \multicolumn{1}{c}{ Outcome } & $\begin{array}{c}\text { MT } \\
(\mathrm{n}=1669)\end{array}$ & $\begin{array}{c}\text { MT+IVT } \\
(\mathrm{n}=1226)\end{array}$ & $\begin{array}{c}\text { OR } \\
(95 \% \mathrm{Cl})\end{array}$ & $\begin{array}{c}\mathrm{p} \\
\text { Value }\end{array}$ \\
\hline $\mathrm{ICH}$ & $296(17.7)$ & $263(21.5)$ & $1.280(1.043-1.571)$ & $\mathbf{0 . 0 1 8}$ \\
\hline $\mathrm{SAH}$ & $98(5.9)$ & $72(5.9)$ & $1.020(0.727-1.432)$ & 0.90 \\
\hline $\mathrm{IVH}$ & $22(1.3)$ & $17(1.4)$ & $1.00(0.991-1.008)$ & 0.932 \\
\hline $\begin{array}{l}\text { Access-site } \\
\text { hemorrhage }\end{array}$ & $12(0.7)$ & $21(1.7)$ & $2.676(1.259-5.686)$ & $\mathbf{0 . 0 1}$ \\
\hline Vasospasm & $22(1.3)$ & $16(1.3)$ & $0.927(0.454-1.889)$ & 0.834 \\
\hline
\end{tabular}

Values represent the number of patients (\%) unless stated otherwise. Boldface type indicates statistical significance.

tion. Hence, this data analysis cannot be compared with trials in which the patients were eligible for both treatment options. The patients in our MT group may or may not have been eligible for IVT. This analysis offers an insight into the safety profile for the MT+IVT group.

Also, we used discharge disposition as a surrogate for the functional outcome, though factors such as patient preference and family support may confound this measure. Again, the outcomes of the MT+IVT group would be influenced by the fact that they arrived in the treatment facility earlier (as they were eligible for IVT) versus the MT-alone group (in which patients may or may not have been eligible for IVT due to arrival at the treatment facility $>4.5$ hours after stroke onset). We did not analyze our patients separately based on whether they initially presented to a community hospital versus a tertiary center (i.e., "drip and ship" vs "mothership" model), though this may also contribute to the outcome. Other limitations include loss of data in exclusion due to missing data fields, interrater coding bias, inability to compare baseline degree of deficit (NIHSS), and extensive variability in ICD-10 codes.

\section{Conclusions}

Although ongoing clinical trials are best suited to address the clinical question of MT alone versus MT+IVT for treatment of AIS due to LVO, real-world data in terms of patient outcomes are essential for gaining a wider perspective. In our analysis of the NIS database, the MT+IVT group had significantly higher rates of minimal disability at the time of hospital discharge compared with the MTalone group, despite a higher rate of ICH. The question of whether to treat patients with MT+IVT rather than MT alone is currently being addressed in ongoing prospective clinical trials (SWIFT-DIRECT [NCT03494920], MR CLEAN-NO IV [ISRCTN80619088], and DIRECTSAFE [NCT03494920]). We look forward to the results of these studies and the progressive improvement in outcomes for AIS patients.

\section{References}

1. Feigin VL, Roth GA, Naghavi M, et al. Global burden of stroke and risk factors in 188 countries, during 1990-2013: a systematic analysis for the Global Burden of Disease Study 2013. Lancet Neurol. 2016;15(9):913-924. 
2. Ovbiagele B, Goldstein LB, Higashida RT, et al. Forecasting the future of stroke in the United States: a policy statement from the American Heart Association and American Stroke Association. Stroke. 2013;44(8):2361-2375.

3. Chen CJ, Ding D, Starke RM, et al. Endovascular vs medical management of acute ischemic stroke. Neurology. 2015; 85(22):1980-1990.

4. Bracard S, Ducrocq X, Mas JL, et al. Mechanical thrombectomy after intravenous alteplase versus alteplase alone after stroke (THRACE): a randomised controlled trial. Lancet Neurol. 2016;15(11):1138-1147.

5. Saver JL, Goyal M, Bonafe A, et al. Stent-retriever thrombectomy after intravenous t-PA vs. t-PA alone in stroke. $N$ Engl J Med. 2015;372(24):2285-2295.

6. Jovin TG, Chamorro A, Cobo E, et al. Thrombectomy within 8 hours after symptom onset in ischemic stroke. $N$ Engl J Med. 2015;372(24):2296-2306.

7. Goyal M, Demchuk AM, Menon BK, et al. Randomized assessment of rapid endovascular treatment of ischemic stroke. N Engl J Med. 2015;372(11):1019-1030.

8. Campbell BC, Mitchell PJ, Kleinig TJ, et al. Endovascular therapy for ischemic stroke with perfusion-imaging selection. N Engl J Med. 2015;372(11):1009-1018.

9. Berkhemer OA, Fransen PS, Beumer D, et al. A randomized trial of intraarterial treatment for acute ischemic stroke. $N$ Engl J Med. 2015;372(1):11-20.

10. Martins SO, Mont'Alverne F, Rebello LC, et al. Thrombectomy for stroke in the public health care system of Brazil. $N$ Engl J Med. 2020;382(24):2316-2326.

11. Coutinho JM, Liebeskind DS, Slater LA, et al. Combined intravenous thrombolysis and thrombectomy vs thrombectomy alone for acute ischemic stroke: a pooled analysis of the SWIFT and STAR studies. JAMA Neurol. 2017;74(3): 268-274.

12. Zi W, Qiu Z, Li F, et al. Effect of endovascular treatment alone vs intravenous alteplase plus endovascular treatment on functional independence in patients with acute ischemic stroke: the DEVT Randomized Clinical Trial. JAMA. 2021; 325(3):234-243.

13. Yang P, Zhang Y, Zhang L, et al. Endovascular thrombectomy with or without intravenous alteplase in acute stroke. $N$ Engl J Med. 2020;382(21):1981-1993.

14. Suzuki K, Matsumaru Y, Takeuchi M, et al. Effect of mechanical thrombectomy without vs with intravenous thrombolysis on functional outcome among patients with acute ischemic stroke: the SKIP Randomized Clinical Trial. JAMA. 2021;325(3):244-253.
15. Goyal M, Menon BK, van Zwam WH, et al. Endovascular thrombectomy after large-vessel ischaemic stroke: a metaanalysis of individual patient data from five randomised trials. Lancet. 2016;387(10029):1723-1731.

16. Albers GW, Marks MP, Kemp S, et al. Thrombectomy for stroke at 6 to 16 hours with selection by perfusion imaging. $N$ Engl J Med. 2018;378(8):708-718.

17. Nogueira RG, Jadhav AP, Haussen DC, et al. Thrombectomy 6 to 24 hours after stroke with a mismatch between deficit and infarct. N Engl J Med. 2018;378(1):11-21.

\section{Disclosures}

The authors report no conflict of interest concerning the materials or methods used in this study or the findings specified in this paper.

\section{Author Contributions}

Conception and design: Guthikonda, Kandregula, Savardekar, Kosty, Cuellar. Acquisition of data: Kandregula. Analysis and interpretation of data: Kandregula, Savardekar, McLarty. Drafting the article: Kandregula, Savardekar, Trosclair. Critically revising the article: Guthikonda, Kandregula, Savardekar, Sharma, Kosty, Trosclair, Cuellar. Reviewed submitted version of manuscript: Guthikonda, Kandregula, Savardekar, Sharma, Kosty, Trosclair, Cuellar. Approved the final version of the manuscript on behalf of all authors: Guthikonda. Statistical analysis: Kandregula, McLarty. Administrative/technical/material support: Guthikonda. Study supervision: Guthikonda, Savardekar, Sharma, Kosty, Cuellar.

\section{Supplemental Information}

Online-Only Content

Supplemental material is available online.

Supplementary Table 1. https://thejns.org/doi/suppl/10.3171/ 2021.4.FOCUS21132.

\section{Correspondence}

Bharat Guthikonda: LSU HSC Shreveport, LA. bguthi@1suhsc. edu. 